\title{
Maternal Style Selectively Shapes Amygdalar Development and Social Behavior in Rats Genetically Prone to High Anxiety
}

\author{
Joshua L. Cohen ${ }^{a}$ Matthew E. Glover ${ }^{a}$ Phyllis C. Pugh ${ }^{a}$ Andrew D. Fant $^{b}$ \\ Rebecca K. Simmons ${ }^{\text {a Huda Akil }}{ }^{c}$ Ilan A. Kerman ${ }^{a}$ Sarah M. Clinton ${ }^{a}$ \\ ${ }^{a}$ Department of Psychiatry and Behavioral Neurobiology, University of Alabama at Birmingham, Birmingham, Ala., \\ ${ }^{b}$ Division of Chemical Biology and Medicinal Chemistry, Eshelman School of Pharmacy, University of North Carolina \\ at Chapel Hill, Chapel Hill, N.C., and 'Molecular and Behavioral Neuroscience Institute, University of Michigan, Ann \\ Arbor, Mich., USA
}

\section{Key Words}

Social interaction - Open field test · Amygdala .

Hippocampus · Microarray · Cross-fostering

\begin{abstract}
The early-life environment critically influences neurodevelopment and later psychological health. To elucidate neural and environmental elements that shape emotional behavior, we developed a rat model of individual differences in temperament and environmental reactivity. We selectively bred rats for high versus low behavioral response to novelty and found that high-reactive (bred high-responder, bHR) rats displayed greater risk-taking, impulsivity and aggression relative to low-reactive (bred low-responder, bLR) rats, which showed high levels of anxiety/depression-like behavior and certain stress vulnerability. The bHR/bLR traits are heritable, but prior work revealed bHR/bLR maternal style differences, with bLR dams showing more maternal attention than bHRs. The present study implemented a cross-fostering paradigm to examine the contribution of maternal behavior to the brain development and emotional behavior of bLR offspring. bLR offspring were reared by biological bLR mothers or fostered to a bLR or bHR mother and then evaluated to determine the effects on the following: (1) developmental gene expression in the hippocampus and amygdala
\end{abstract}

and (2) adult anxiety/depression-like behavior. Genomewide expression profiling showed that cross-fostering bLR rats to bHR mothers shifted developmental gene expression in the amygdala (but not hippocampus), reduced adult anxiety and enhanced social interaction. Our findings illustrate how an early-life manipulation such as cross-fostering changes the brain's developmental trajectory and ultimately impacts adult behavior. Moreover, while earlier studies highlighted hippocampal differences contributing to the bHR/bLR phenotypes, our results point to a role of the amygdala as well. Future work will pursue genetic and cellular mechanisms within the amygdala that contribute to bHR/ bLR behavior either at baseline or following environmental manipulations.

(c) 2015 S. Karger AG, Basel

\section{Introduction}

The early-life environment critically influences brain development and subsequent psychological health, with some individuals being particularly vulnerable to psycho-

Joshua L. Cohen and Matthew E. Glover contributed equally to this work.

\section{KARGER 125}

C 2015 S. Karger AG, Basel

$0378-5866 / 15 / 0373-0203 \$ 39.50 / 0$

E-Mail karger@karger.com

www.karger.com/dne
Sarah M. Clinton

1720 7th Avenue South, SC 745

Birmingham, AL 35294 (USA)

E-Mail clintons@uab.edu 
pathology due to combined genetic predisposition and environmental risks $[1,2]$. Children of depressed mothers are at greater risk for psychological problems than children of nondepressed mothers [3, 4], although early intervention mitigates their risk for emotional disorders. For instance, epidemiological studies report that children of depressed mothers manifest fewer emotional problems when enrolled in childcare from an early age [5], suggesting that stimulation of exposure to a new environment during development boosts their emotional health. Rodent studies echo these findings, showing that short periods of novelty exposure during the early postnatal period (independent of changes in maternal care) improve anxiety-like behavior and hypothalamic-pituitary-adrenal (HPA) stress response [6]. Together, these studies highlight how genetic liability and experience interact to shape neurodevelopment, biobehavioral responses to stress and life-long emotional health [7].

To elucidate molecular changes in the developing brain that produce an anxious phenotype, we developed a rat model of individual differences in emotionality by selectively breeding Sprague-Dawley rats for disparate response to novelty [8]. Rats bred for high response to novelty (bred high-responder, bHR, rats) vigorously explore new environments, exhibit greater risk-taking, impulsivity, aggression, and proclivity to addictive behavior $[9,10]$ compared to bred low-responder (bLR) rats, which show high levels of spontaneous anxiety and depressive-like behavior [8, 11-14]. The apparent cosegregation of high/ low novelty reactivity and these other behavioral characteristics may be due to selective breeding or could be secondary to genetic drift [15], although it is worth noting that many of these behaviors appear in purchased (nonselectively bred) rats screened for high versus low response to novelty $[16,17]$.

A valuable feature of the bred lines is their phenotypic predictability, which allows us to foretell whether an animal will display bHR/bLR traits from birth throughout life. This shows us that the bHR/bLR biobehavioral phenotypes are present in early postnatal life $[11,18]$ and are associated with distinct patterns of hippocampal development [18]. Furthermore, we found that while the bHR/bLR traits are heritable, their phenotypes (particularly bLRs) are also modifiable by environmental factors $[11,12,14]$. Because we know that bHR/ bLR dams exhibit distinct maternal styles [19, 20], and given the profound effect of maternal behavior on rodent neurodevelopment, the HPA axis and emotional behavior [21], the present study examined the contribution of maternal behavior to brain development and be- havior in the high anxiety/depression-prone bLR offspring. An important advance of this study is that it not only illustrates the impact of distinct maternal styles on the adult brain and behavior but also tracks developmental changes in the limbic brain when the early-life experience is ongoing.

Thus, we implemented a cross-fostering paradigm where bLR offspring were raised under the following maternal conditions: (1) reared by biological bLR mothers, (2) fostered to bLR mothers or (3) cross-fostered to bHR mothers. We focused on the bLR line since prior studies found that the bHRs are resistant to many environmental manipulations, including cross-fostering and early-life stress $[8,14]$. The first experiment examined the impact of cross-fostering on anxiety/depression-like behavior and social behavior of adult bLR males. This experiment included a bHR control group (bHRs raised by their biological mothers) to provide a benchmark to highlight typical bHR/bLR behavioral differences. A parallel microarray study examined maternally regulated gene expression changes in the developing amygdala and hippocampus of bLR male offspring to identify neurobiological changes underlying maternal influences on the emotional behavior of bLRs.

\section{Materials and Methods}

All experiments were approved by the University Committee on the Use and Care of Animals at the University of Alabama at Birmingham (UAB), where the behavioral studies and brain tissue harvesting were conducted. All work was conducted in accordance with the National Institute of Health (NIH) Guide for the Care and Use of Laboratory Animals, as dictated by the National Research Council in 1996.

\section{Animals}

Adult male and female bHR/bLR Sprague-Dawley rats from the 30th generation of Dr. Akil's colony at the University of Michigan were shipped to Dr. Clinton's laboratory at UAB where they were bred for the present experiments. We previously described our breeding strategy, noting that our breeding paradigm leads to $1 \%$ inbreeding per generation [8]. Thus, rats from the present study were $31 \%$ inbred since they were taken from the 31 st generation. Animal facilities were kept at $21-23^{\circ} \mathrm{C}$ at $50-55 \%$ humidity and all rats were group-housed in a 12:12 light-dark cycle (lights on/off at 6 a.m./6 p.m.) with food and water available ad libitum. Only male offspring were studied in the behavioral and microarray experiments described below.

\section{Cross-Fostering Paradigm}

For experiment 1 , adult male/female pairs $(n=5$ bHR pairs; $n=$ 11 bLR pairs) were mated. At birth, bLR litters were randomly assigned to be raised as follows: (1) reared by their biological bLR mother (bLR control), (2) fostered to another bLR mother (bLR- 
bLR) or (3) cross-fostered to a bHR mother (bLR-bHR). The bHR litters used for behavioral experiments were reared by biological bHR mothers (bHR control). Offspring were weaned on postnatal day 21 (P21) and only males were kept for a behavioral test battery that commenced in adulthood (P75).

\section{Behavioral Testing}

All behavior was recorded using a computerized analysis program (Ethovision ${ }^{\circledR}$ XT 8.0; Noldus, Wageningen, The Netherlands), and all testing was conducted under dim light (30 lx) between 8:00 and 11:30 a.m. Detailed descriptions of our test paradigms can be seen below.

\section{Open Field}

The open field test (OFT) was conducted in a $100 \times 100 \times 50 \mathrm{~cm}$ black Plexiglas box with a black floor as described [22]. At the beginning of the test, a rat was placed in a corner of the box and was permitted to explore the apparatus for $5 \mathrm{~min}$. The latency to enter the center of the OF, the amount of time spent and distance traveled in the center, sides and corners of the apparatus were quantified utilizing Ethovision XT 8.0 videotracking software (Noldus) set up with a digital video camera. The periphery was defined by a $20-\mathrm{cm}$ zone around the edge of the $\mathrm{OF}$ arena that was further subdivided into mutually exclusive side $(20 \times 60 \mathrm{~cm})$ and corner $(20 \times$ $20 \mathrm{~cm}$ ) zones. A trained observer who was blinded to the experimental groups manually assessed grooming and rearing behavior and stress-induced defecation using a computerized system provided in the software.

\section{Elevated Plus Maze}

The elevated plus maze (EPM) test was conducted as described [22] in a black Plexiglas EPM consisting of four elevated arms (70 $\mathrm{cm}$ from the floor, $45 \mathrm{~cm}$ long, $12 \mathrm{~cm}$ wide) arranged in a cross. Two opposite arms were enclosed by $45-\mathrm{cm}$-high walls (lighting 3-5 lx), and the remaining arms were open (lighting $30 \mathrm{~lx}$ ). To start the test the rats were placed in a central square platform facing the same closed arm at the intersection of the open and closed arms, providing access to all arms. Testing was conducted under dim light (30 lx) between 8:00 and 11:30 a.m. and recorded using the Ethovision videotracking system to assess the latency to enter the open arms, the amount of time spent in the open arms, closed arms and central square and the total distance traveled over the course of the 5-min test. An animal was considered to be in the open arm when the rat's body fully crossed out of the central square onto an open arm platform. A trained observer who was blinded to the experimental groups manually assessed stress-induced defecation.

\section{Novelty-Suppressed Feeding}

The novelty-suppressed feeding (NSF) test was conducted in the OF apparatus. The rats were deprived of food overnight prior to testing. At the beginning of the 10-min test, a rat chow food pellet was placed in the center of the OF. A rat was placed in the periphery of the arena and permitted to freely explore for $10 \mathrm{~min}$. Latencies to sniff, touch and eat the food pellet were measured by a trained observer blinded to experimental groups. The rats were able to remove the food pellet from the center of the arena. The movement of the animals was assessed by Ethovision XT 8.0 software. Food pellet weight was manually measured before and after each session.

Maternal Care, Brain Development and Anxiety

\section{Social Interaction}

A social interaction test was conducted as described [22] using a 3-day procedure where experimental animals spent $10 \mathrm{~min}$ per day in a clear Plexiglas chamber $(45 \times 45 \times 60 \mathrm{~cm}$ Noldus PhenoTyper $^{\circledR}$ Box $)$ containing a smaller Plexiglas 'interaction box' $(10 \times$ $10 \times 8 \mathrm{~cm}$ ) that held a novel stimulus rat. On test day 1 , the rats were placed in the chamber with an empty interaction box (novel object). On test day 2, a novel male rat was placed in the interaction box shortly before the experimental rat was introduced. On test day 3, a novel female rat was placed in the interaction box shortly before the experimental rat was introduced. In each test, a blinded, trained observer scored grooming behavior $[23,24]$ and stress-induced defecation $[25,26]$ as secondary measures of rodent anxiety.

\section{Forced Swim Test}

The forced swim test (FST) was conducted as described [22] in a Plexiglas cylinder ( $40 \mathrm{~cm}$ high $\times 40 \mathrm{~cm}$ diameter) containing water $30 \mathrm{~cm}$ deep $\left(25^{\circ} \mathrm{C}\right)$. On FST day 1, the rats were placed ( $1 \mathrm{rat} /$ cylinder) in the water for $15 \mathrm{~min}$ (pretest phase); $24 \mathrm{~h}$ later the rats were returned to the water-filled cylinder and videotaped for $5 \mathrm{~min}$ (test phase). The water was changed after every swim session so that each rat swam in clean water. A trained observer blinded to treatment scored the FST immobility (floating) time using a computer program for ethological analysis (Observer; Noldus). The rats were considered immobile when they exhibited no activity other than that required to keep their heads above water. We chose to focus on the immobility measure since it is classically considered an indicator of 'behavioral despair' and depression-like behavior, and it can be clearly defined and easily distinguishable from active coping measures such as swimming and climbing, which are sometimes difficult to reliably distinguish across experimental observers. Data are presented as time immobile as the percentage of total time during the 5-min day 2 test.

\section{Tissue Collection, RNA Extraction and Genome-Wide} Expression Profiling

For experiment 2, another group of male/female rats were mated and the cross-fostering procedure was repeated. Brain tissue was collected from bLR males that were raised as follows: (1) by biological bLR mothers (bLR control), (2) by bLR foster mothers (bLR-bLR fostered) or (3) by bHR foster mothers (bLR-bHR crossfostered). The animals were sacrificed by rapid decapitation at 4 time points: P7, P14, P21, and P75 ( $\mathrm{n}=5$ /group/time point for microarray experiments, $n=5$ /group/time point for the qPCR experiments described below). The brains were removed, flash frozen and stored at $-80^{\circ} \mathrm{C}$ until further processing. Brain tissue punches were collected from the hippocampus and amygdala as we previously described [27], placed in RNAlater (Qiagen, Valencia, Calif., USA) and stored at $-20^{\circ} \mathrm{C}$.

Microarray Analysis

Tissue punches ( $\mathrm{n}=5$ /group/time point/brain region) were shipped to Arraystar (Rockville, Md., USA) for microarray processing on NimbleGen rat gene expression arrays of $12 \times 135 \mathrm{~K}$ (26,419 target genes, 5 probes/target). RNA from each sample was isolated, purified and processed for microarray analysis according to the manufacturer's protocol (NimbleGen Systems, Inc., Madison, Wisc., USA). Slides were later scanned at $5 \mu \mathrm{m} /$ 
pixel resolution using an Axon GenePix 4000B scanner (Molecular Devices Corporation) piloted by GenePix Pro 6.0 software (Axon). Scanned images were imported into NimbleScan software (version 2.5) for grid alignment and expression data analysis. Expression data were normalized through quantile normalization and the robust multichip average algorithm included in the NimbleScan software. The probe and gene level files were generated after normalization.

Subsequent data analysis was performed using GeneSpring GX v12.6 software (Agilent, Santa Clara, Calif., USA). Probes with a raw expression value less than 50 for any sample were excluded from the analysis. Samples were filtered to exclude probes designated 'predicted mRNA model', as determined by the most recent RefSeq database annotations. Following experimental grouping (bLR-bHR cross-foster, or bLR-bLR foster, vs. bLR control at each time point within each brain region), genes were considered statistically significant and included in downstream analyses if they displayed a fold change $\geq 1.5$, with a $\mathrm{p}$ value $<0.05$ following multiple test correction (Bonferroni family-wise error rate, FWER). Probes in the P75 comparison were selected for k-means partitional clustering if they met these criteria. Gene ontology (GO) analysis identified the enrichment of functionally related genes within each cluster ( $\mathrm{p}$ value $<0.05$ following Benjamini-Yekutieli false discovery rate, FDR, correction). All genes and transcripts discussed in this paper are referenced by the Rattus norvegicus gene symbol.

Quantitative Real-Time PCR Confirmation Studies

Select genes identified in the microarray analysis were verified using quantitative real-time PCR (qRT-PCR). Total RNA was extracted and purified from a separate set of amygdala tissue punches from P75 bLR control and bLR-bHR fostered male offspring ( $\mathrm{n}=5$ /group). We performed qRT-PCR as described [18] on a StepOne Plus (Applied Biosystems, Grand Island, N.Y., USA). The following TaqMan assay probes were used: Ywhaq (RefSeq: NM_013053.1; Taqman probe ID: Rn00820723_g1), Nptxr (NM_030841.3; Rn00578183_m1), Camk2a (NM_012920.1; Rn01258147_m1), Dlg3 (NM_031639.1; Rn00581244_m1), Dlg4 (NM_019621.1; Rn00571479_m1), and Lppr2 (NM_001005881.1; Rn00460732_g1). TaqMan probes for Cx3cl1 (NM_134455.1; Rn00593186_m1), Glul (NM_017073.3; Rn01483107_m1) and Syt1 (NM_001033680.2; Rn00436862_m1) were used as housekeeping genes.

\section{Statistical Analyses}

Statistical analyses were performed using GraphPad Prism 5 (GraphPad Software, La Jolla, Calif., USA). To control for the possibility of litter effects, data from individual animals from each experimental group were averaged and these were used in subsequent statistical analyses. Because only bLR groups were subjected to different fostering conditions, with the bHR controls raised only in the biological mother condition to serve as a 'benchmark' to highlight bHR/bLR differences, our design required a one-way ANOVA to examine behavioral differences among bLR groups in the OF, EPM, NSF, and FST. In the social interaction test, we used a two-way ANOVA, with bLR maternal care condition and social stimulus condition as independent factors. Fisher's LSD post hoc analyses were used when appropriate (for all tests, $\alpha=0.05$ ). Data analysis methods for the microarray experiment are noted above.

\section{Results}

Cross-Fostering bLR Pups to bHR Mothers

Improved Select Measures of Adult Anxiety-Like

Behavior and Social Interaction but Did Not

Impact Depression-Like Behavior in the FST

Adult bLR offspring raised by bLR biological mothers, bLR or bHR foster mothers (as well as bHR controls raised by their biological mothers) were evaluated in multiple tests of anxiety-and depression-like behavior. In the OFT, there was a main effect of group on center time $\left(\mathrm{F}_{3,15}=16.37, \mathrm{p}<0.001\right.$; fig. 1a), time spent grooming $\left(\mathrm{F}_{3,15}=4.05, \mathrm{p}<0.05\right.$; fig. $\left.1 \mathrm{c}\right)$ and total distance traveled during the OFT $\left(\mathrm{F}_{3,15}=83.33, \mathrm{p}<0.0001\right.$; fig. 1e). Many of these effects were due to genotypic differences, with post hoc analysis showing that bHR controls spent more time in the center of the OF $(\mathrm{p}<0.001)$ and overall greater activity $(\mathrm{p}<0.0001)$ than all bLR groups. bHR controls also spent less time grooming than bLRs raised by biological mothers and bLR foster mothers $(\mathrm{p}<0.05)$. Crossfostering subtly decreased anxiety-like behavior of bLR offspring in the OF, with bLR offspring raised by bHR mothers showing increased locomotion $(\mathrm{p}<0.05)$ relative to bLRs raised by biological mothers and bLR foster mothers and trending towards less grooming $(\mathrm{p}=0.07)$ relative to bLRs raised by biological mothers. There was a trend for bLR-bHR fostered offspring to differ from bLR-bLR fostered offspring on these measures.

There were similar effects in the EPM, with a main effect of group on open arm time $\left(\mathrm{F}_{3,15}=6.53, \mathrm{p}<0.01\right.$; fig. $1 b)$, stress-induced defecation $\left(\mathrm{F}_{3,15}=15.25, \mathrm{p}<\right.$ 0.001; fig. 1d) and total distance traveled in the EPM $\left(\mathrm{F}_{3,15}=32.45, \mathrm{p}<0.0001\right.$; fig. 1f). Again, many of the effects were due to genotypic differences, with post hoc analysis showing that bHR controls spent more time in the open arms of the EPM ( $<<0.01)$ and overall greater activity $(\mathrm{p}<0.0001)$ than bLRs. bHR controls also showed less stress-induced defecation in the EPM than bLR controls and bLRs raised by bLR foster mothers $(\mathrm{p}<0.01)$. Cross-fostering subtly decreased anxiety-like behavior of bLR offspring in the EPM, with bLR offspring raised by bHR mothers showing less stress-induced defecation than bLR controls and bLRs raised by bLR foster mothers $(\mathrm{p}<0.01)$.

In the social interaction test, there was a main effect of experimental group $\left(\mathrm{F}_{3,47}=13.4, \mathrm{p}<0.001\right)$ but no effect of social stimulus (novel object/male/female) and no group $\times$ stimulus interaction on the number of visits to the interaction box (fig. 2a). There was a genotypic difference, with bHR controls showing high levels of interac- 


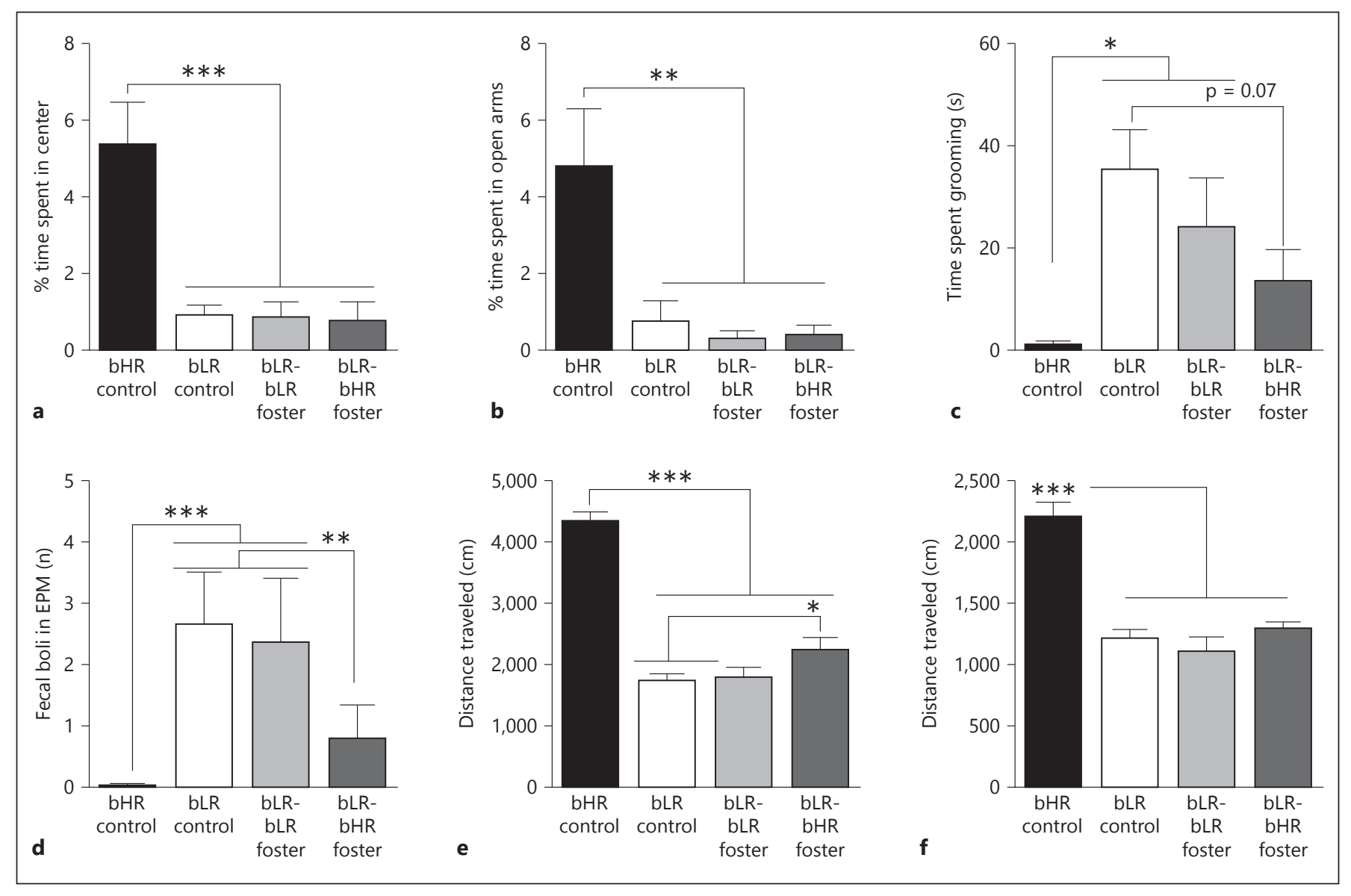

Fig. 1. Cross-fostering bLR offspring to bHR mothers subtly reduced adult anxiety-like behavior in the OFT and EPM. a Crossfostering did not affect OF center time of bLR offspring, with bLRbHR cross-fostered offspring spending similarly low amounts of time in the center as bLR controls and bLR-bLR fostered rats. The bHR controls spent more time in the center than all bLR groups. b Likewise, in the EPM, cross-fostering did not affect time in open arms of bLR offspring, with bLR-bHR fostered rats spending as little time in the open arms as bLR controls and bLR-bLR fostered animals. The bHR controls spent significantly more time in the open arms. c Cross-fostered bLR offspring exhibited less grooming compared to bLR controls, indicating less anxiety. bHRs groomed less than bLR controls and bLR-bLR fostered offspring (but did not differ from cross-fostered bLRs). d Cross-fostered bLRs showed

tion with the novel object, male stimulus rat and female stimulus rat compared to bLR groups $(\mathrm{p}<0.01$ for each comparison). There was also a clear effect of cross-fostering, with bLRs raised by bHR mothers showing increased interactions with a novel object, male stimulus rat and female stimulus rat compared to the bLR controls and bLRs raised by bLR mothers ( $\mathrm{p}<0.05$ for each comparison). There was also a main effect of group $\left(\mathrm{F}_{3,47}=23.04\right.$, $\mathrm{p}<0.001$ ) but no effect of social stimulus (novel object/

Maternal Care, Brain Development and Anxiety less stress-induced defecation in the EPM relative to bLR controls, another indicator of reduced anxiety. bHR controls showed less defecation compared to bLR controls and bLR-bLR fostered rats but did not differ from bLR offspring cross-fostered to bHR mothers. e In the OF, bHR controls showed greater overall activity compared to the 3 bLR groups, although bLR offspring cross-fostered to bHR mothers displayed a slight but significant increase in novelty-induced activity compared to bLR controls that were raised by their biological bLR mothers. $f$ In the EPM, bHR controls again showed greater overall activity compared to the 3 bLR groups. Maternal care condition did not affect total activity of bLR offspring in the EPM. Data represent means \pm SEM. ${ }^{*} \mathrm{p}<0.05 ;{ }^{* *} \mathrm{p}<0.01$; $* * * \mathrm{p}<0.001$.

male/female) and no group $\times$ stimulus interaction on stress-induced defecation in the social interaction test (fig. 2b). There was a genotypic difference, with bHRs showing less defecation than all bLR groups $(\mathrm{p}<0.001$ for each comparison). Cross-fostering reduced stress-induced defecation of bLRs, with the bHR-fostered bLR groups showing significantly less defecation than bLR controls and bLR-bLR fostered offspring ( $p<0.01$ for each comparison). 


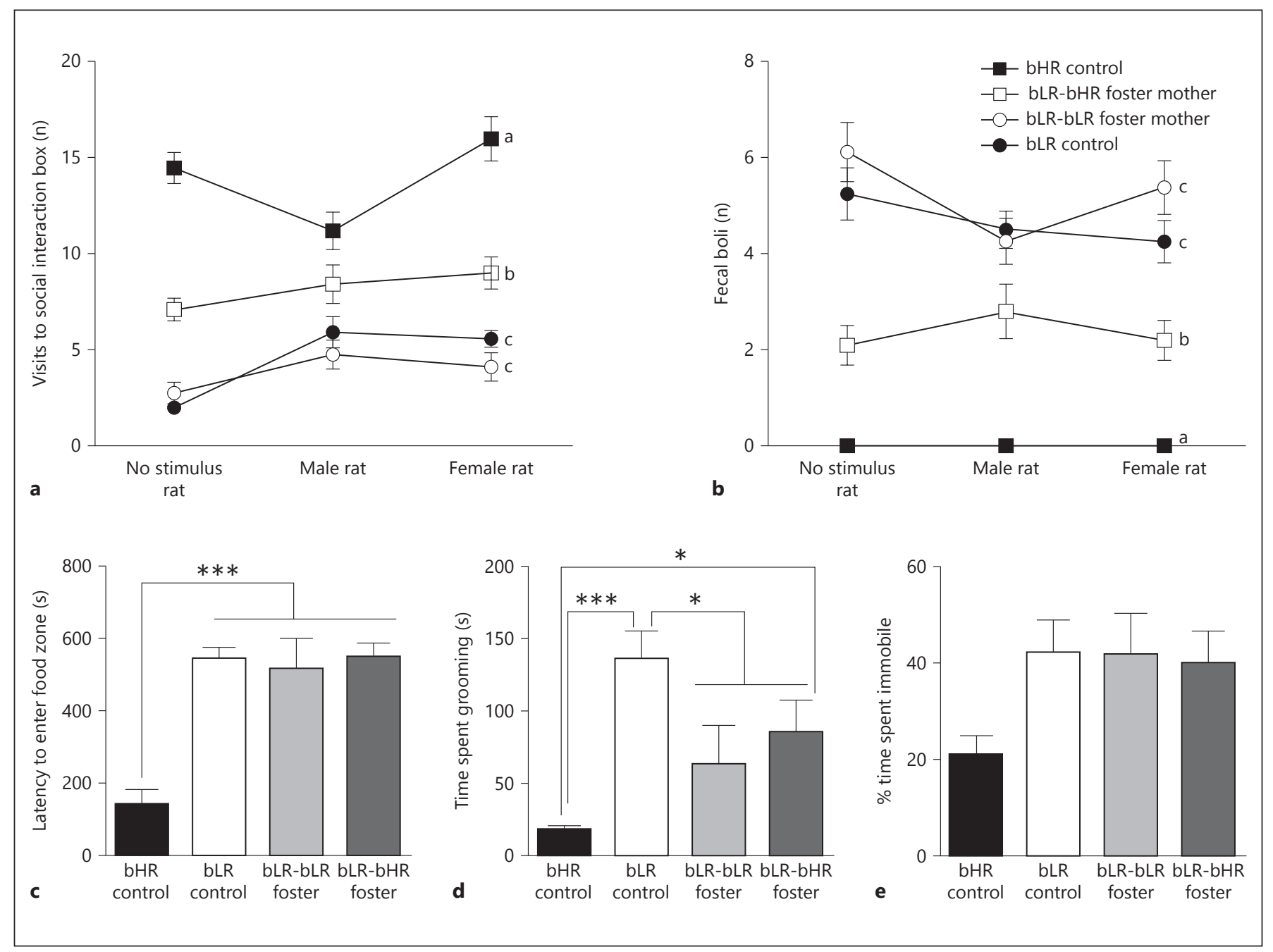

Fig. 2. Cross-fostered bLRs showed enhanced social interaction and subtly reduced anxiety in the NSF test but no change in FST immobility. a In a social interaction test, bHR controls showed much higher levels of interaction with a novel object, a male stimulus rat and a female stimulus rat compared to bLR controls and bLR-bLR fostered offspring (indicated by symbol a vs. c). Crossfostering significantly enhanced the social interaction of bLRs, with the bLR-bHR fostered group showing more interaction compared to the 2 other bLR groups (indicated by symbol b vs. c). b The bHR controls showed much less stress-induced defecation in the social interaction test compared to the 3 bLR groups (indicated by symbol a vs. symbols b and c). Cross-fostered bLRs displayed less stress-induced defecation (indicating less anxiety) dur-

In the NSF task, there was a main effect of group on latency to enter the food zone $\left(\mathrm{F}_{3,15}=22.43, \mathrm{p}<0.0001\right.$; fig. 2c) and a main effect of group on time spent grooming $\left(\mathrm{F}_{3,15}=11.64, \mathrm{p}<0.001\right.$; fig. $\left.2 \mathrm{~d}\right)$. The bHR control group showed the shortest latency to enter the food zone compared to all three bLR groups ( $p<0.0001$ for ing the test relative to bLR controls and bLR-bLR fostered offspring (indicated by symbol b vs. c). c In the NSF test, bHRs had a shorter latency to approach the food zone compared to all 3 bLR groups; cross-fostering did not impact this measure as all 3 bLR groups showed similarly high latencies. $\mathbf{d}$ bLR controls spent more time grooming during the NSF test than all other groups. There was a general effect of fostering on this measure as both bLR-bLR fostered and bLR-bHR fostered groups showed significantly less grooming in the NSF compared to bLR controls. e In the FST, bHR controls showed low immobility levels compared to the 3 bLR groups; there was no effect of maternal care condition on FST immobility of bLR offspring. Data represent means \pm SEM. ${ }^{*} p<0.05$; ${ }^{* * *} \mathrm{p}<0.001$.

all comparisons) and spent less time grooming than bLR controls $(p<0.05)$. There was a general effect of fostering on grooming of bLRs in the NSF since both bLR-bLR fostered and bLR-bHR cross-fostered offspring showed less grouping than bLR controls $(\mathrm{p}<0.05$ for each comparison). 
Finally, in the FST, while there was no effect of group on immobility time (fig. 2e), the bHR group spent the least amount of time immobile. Maternal care condition did not influence FST immobility of bLRs since all three bLR groups displayed similarly high immobility levels.

Cross-Fostering Shifts Developmental Gene Expression in the bLR Amygdala but Not Hippocampus

Gene expression analysis (fold change $\geq 1.5, \mathrm{p}<0.05$, Bonferroni FWER) revealed robust developmental gene expression changes within the amygdala, but not the hippocampus, of bLR offspring that were cross-fostered to bHR dams compared to bLR controls (bLR-bHR crossfostered/bLR control; fig. 3a). Notably, we found extraordinarily few gene expression differences in the amygdala or hippocampus of bLR offspring fostered to bLR mothers (bLR-bLR fostered/bLR control). Although cross-fostering had little effect on bLR amygdalar gene expression at P7 and P14, numerous gene changes appeared at P21 and persisted into adulthood, with the majority of altered genes being upregulated in bLR-bHR cross-fostered offspring compared to bLR controls (fig. 3b). After applying the FDR correction, no genes were found to be differentially expressed in the hippocampus at any time point. A complete list of genes significantly altered at each time point can be found in online supplementary tables 1-3 (for all online suppl. material, see www.karger.com/ doi/10.1159/000374108).

Our next analysis used partitional clustering to examine the amygdala gene expression data, grouping genes altered by cross-fostering based on developmental expression patterns. Partitional clustering of the 103 genes in the P75 amygdala data set assigned genes to four clusters based on the slope of the expression pattern of genes across time (differential distance metric; shown in fig. 3c). GO analysis identified functionally related genes within the four gene clusters and found significant GO term enrichment for cluster 1 (GO:0007611, learning and memory, $\mathrm{p}=7.44 \times 10^{-5}$; GO:0050890, cognition, $\mathrm{p}=7.88 \times$ $10^{-5}$ ) and cluster 3 (GO:0016192, vesicle-mediated transport, $\mathrm{p}=1.26 \times 10^{-5} ; \mathrm{p}<0.05$ after multiple test corrections; fig. 3c). Several of the learning and memory annotated transcripts from cluster 1 were chosen for qRT-PCR confirmation; 6 genes were confirmed to be upregulated in the amygdala of adult cross-fostered bLR animals (Ywhag, Nptxr, CamkIIa, Dlg3, Dlg4, and LPPR2) versus bLR controls (table 1). Pathway analysis was performed using the 103 genes in the P75 amygdala data set, revealing 123 pathways that were significantly altered by crossfostering ( $p<0.05$ after Benjamini-Yekutieli FDR correc-

Maternal Care, Brain Development and Anxiety tion). The top 10 pathways by $\mathrm{p}$ value are listed in table 2 , with multiple involving genes related to synaptic signaling, glutamatergic signaling, adrenergic signaling, and apoptosis.

\section{Discussion}

The early family life environment shapes human neurodevelopment and behavioral outcomes well into adulthood $[28,29]$, and early-life events have been shown to predispose or protect individuals from developing later psychopathology. In rodents, maternal style shapes the offspring's neuroendocrine stress reactivity and anxiety/ depression-like behavior $[21,30,31]$. We previously reported distinct maternal styles in bHR/bLR dams $[19,20]$ and now show that cross-fostering bLR offspring to bHR mothers shifts their adult phenotype, leading to greater social interaction and subtly reduced anxiety-like behavior. These effects are accompanied by gene expression changes in the amygdala that emerge in early postnatal life (when the cross-fostering experience is ongoing) and persist through adulthood. There is a growing appreciation that emotional disorders involve a developmental component, and that changes in the developing brain can set the stage for later emotional health [32]. The present study illustrates how an early-life manipulation such as cross-fostering changes the brain's developmental trajectory, ultimately impacting adult behavior.

We previously found that $\mathrm{bHR} / \mathrm{bLR}$ rats exhibit baseline differences in hippocampal development during the first 2 postnatal weeks [18] and that manipulating the hippocampal development of bLRs via early-life injection of fibroblast growth factor 2 reduced their adult anxietylike behavior [33]. It was therefore surprising to find that cross-fostering improved aspects of anxiety behavior of bLRs (grooming in the OF, stress-induced defecation in the EPM and social interaction test) without altering hippocampal gene expression. Cross-fostering did markedly alter gene expression in the bLR amygdala, so it may be that certain aspects of the behavior of bLRs are more closely linked to the hippocampus while others are associated with the amygdala. For example, cross-fostering had the greatest impact on the social interaction of bLRs, with only subtle improvements on anxiety measures (grooming and stress-induced defecation) and no effect on FST immobility. The amygdala plays a well-recognized role in regulating anxiety and fear behavior [34-36] but also mediates social behavior. For instance, stimulating basolateral amygdala terminals in the ventral hippocampus re- 


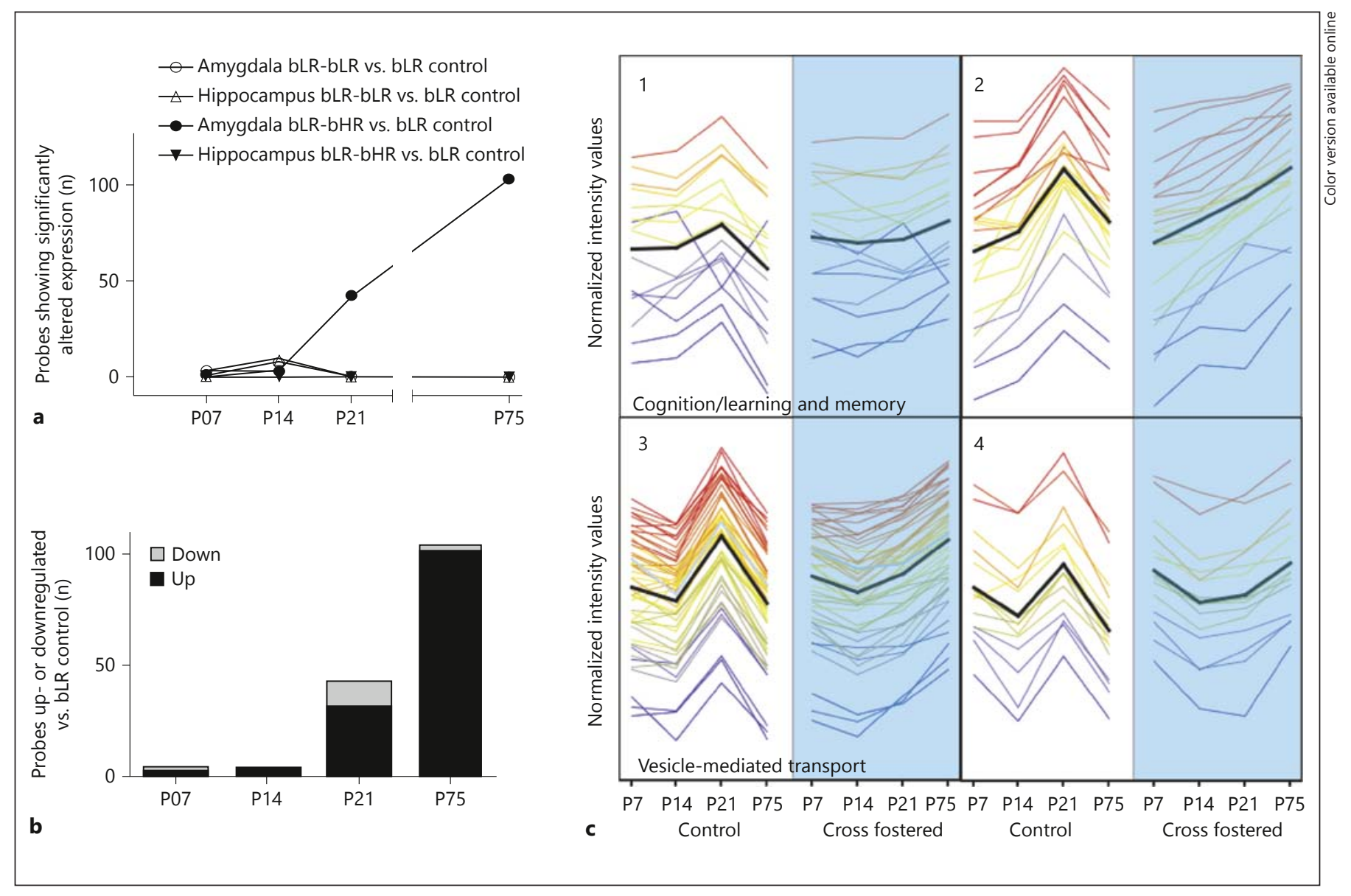

Fig. 3. Cross-fostering bLR offspring to bHR mothers induced marked developmental gene expression changes in the amygdala but not the hippocampus. At birth, bLR pups were assigned to 1 of 3 maternal conditions and raised by their biological mother (bLR control), a bLR foster mother (bLR-bLR fostered group) or a bHR foster mother (bLR-bHR cross-fostered group). Hippocampal and amygdalar tissue punches were collected from brains harvested at P7, P14, P21, and P75 for genome-wide expression profiling. Genes were considered to be significantly altered by maternal care condition if they showed a fold change cutoff $\geq 1.5$ and a $\mathrm{p}$ value $<0.05$ following Bonferroni FWER multiple test correction. a Microarray analysis revealed robust developmental gene expression changes in the amygdala, but not the hippocampus, of bLR offspring that were raised by bHR foster mothers compared to bLR controls. There were minimal gene expression changes in the amygdala and hippocampus of bLR-bLR fostered offspring. $\mathbf{b}$ The number of genes significantly up- or downregulated in the devel-

duced social interaction, while inhibition of these terminals increased social interaction [37]. Another study demonstrated that direct stimulation of distinct subpopulations of amygdalar neurons controlled distinct aspects of self-grooming, social, and asocial behavior [38]. Thus, the bLR cross-fostering paradigm provides a novel mod- oping amygdala of bLR-bHR fostered offspring. c K-means partitional clustering examined 103 differentially expressed genes in the P75 amygdala data set. Genes were assigned to 4 clusters based on similarity of expression profiles (shown in normalized intensity values on the $y$-axis). Developmental gene expression patterns from P7 through P75 are shown from left to right for bLR controls (white background, left; color in online version only) and bLRbHR cross-fostered offspring (blue background, right). Time points are shown with equidistant spacing despite different intervals between some points. Each line represents a gene and the line color reflects normalized intensity value at P75 in the cross-fostered group (far right point in each cluster). The black line indicates mean expression profile for each cluster. GO analysis of transcripts within each cluster revealed significant enrichment for specific GO terms in cluster 1 (GO:0007611, learning and memory, $\mathrm{p}=7.44 \times 10^{-5} ;$ GO:0050890, cognition, $\mathrm{p}=7.88 \times 10^{-5}$ ) and cluster 3 (GO:0016192, vesicle-mediated transport, $\mathrm{p}=1.26 \times 10^{-5}$ ).

el to study the development of amygdalar circuits involved in social behavior and the environmental factors that influence its formation.

The amygdalar gene expression changes triggered by cross-fostering were not apparent until P21 and were most pronounced in adulthood (P75). Importantly, these 
Table 1. Genes confirmed by qPCR as differentially expressed in bLR cross-fostered to bHR versus control bLR in the amygdala of adult animals (P75)

\begin{tabular}{lll}
\hline Gene symbol & Fold change by PCR & Fold change by microarray \\
\hline Ywhag & 1.8 & 4.48 \\
Nptxr & 1.31 & 4.96 \\
CamkIIa & 1.69 & 2.41 \\
Dlg3 & 3.48 & 2.51 \\
Dlg4 & 1.22 & 3.25 \\
Lppr2 & 1.49 & 2.78 \\
\hline
\end{tabular}

Table 2. Top hits of pathway analysis

\begin{tabular}{|c|c|c|c|c|c|c|}
\hline Rank & Pathway & $\begin{array}{l}\log \\
\text { ( } p \text { value) }\end{array}$ & $\mathrm{p}$ value & $\begin{array}{l}\text { Corrected } \\
\text { p value }\end{array}$ & Ratio & Genes \\
\hline 1 & $\begin{array}{l}\text { Neurophysiological process: synaptic vesicle } \\
\text { fusion and recycling in nerve terminals }\end{array}$ & 7.206 & $6.22 \mathrm{E}-08$ & $2.69 \mathrm{E}-05$ & $7 / 52$ & $\begin{array}{l}\text { PIPKI- } \gamma \text {, Synaptotagmin I, Csp, } \\
\text { SLC17A7, MUNC18, SYP, NSF }\end{array}$ \\
\hline 2 & Neuroprotective action of lithium & 6.616 & $2.42 \mathrm{E}-07$ & $5.23 \mathrm{E}-05$ & $7 / 63$ & $\begin{array}{l}\text { PSD-95 (Dlg-4), MKK7 (MAP2K7), } \\
\text { CDK5R1 (p25), CDK5R1 (p35), Dsh, } \\
\text { ATF-2, Amyloid- } \beta\end{array}$ \\
\hline 3 & Development: A1 receptor signaling & 5.78 & $1.66 \mathrm{E}-06$ & $2.39 \mathrm{E}-04$ & $6 / 53$ & $\begin{array}{l}\text { Adenosine A1 receptor, AKT (PKB), } \\
\text { ATF-2, G-protein a-I family, } \\
\text { PKA-cat, PKA-reg }\end{array}$ \\
\hline 4 & Nicotine signaling in glutamatergic neurons & 5.53 & $2.95 \mathrm{E}-06$ & 3.19E-04 & $5 / 33$ & $\begin{array}{l}\text { Adenosine A1 receptor, CaMK IIa, } \\
\text { G-protein } \alpha \text {-I family, PKA-cat, } \\
\text { PKA reg }\end{array}$ \\
\hline 5 & $\begin{array}{l}\text { Neurophysiological process: constitutive and } \\
\text { regulated NMDA receptor trafficking }\end{array}$ & 5.332 & $4.66 \mathrm{E}-06$ & $4.02 \mathrm{E}-04$ & $6 / 63$ & $\begin{array}{l}\text { CDK5R1 (p35), G-protein a-I family, } \\
\text { PKA-cat, PKA-reg, PSD-95 (Dlg-4), } \\
\text { SAP } 102 \text { (Dlg3) }\end{array}$ \\
\hline 6 & Apoptosis and survival: BAD phosphorylation & 4.997 & $1.01 \mathrm{E}-05$ & $7.00 \mathrm{E}-04$ & $5 / 42$ & $\begin{array}{l}\text { 14-3-3, AKT (PKB), Bcl-XL, } \\
\text { PKA-cat, PKA-reg }\end{array}$ \\
\hline 7 & Development: A2A receptor signaling & 4.945 & $1.13 \mathrm{E}-05$ & $7.00 \mathrm{E}-04$ & $5 / 43$ & $\begin{array}{l}\text { AKT (PKB), ATF-2, MKK7 } \\
\text { (MAP2K7), PKA-cat, PKA-reg }\end{array}$ \\
\hline 8 & $\begin{array}{l}\text { Apoptosis and survival } \beta 2 \text { adrenergic receptor } \\
\text { antiapoptotic action }\end{array}$ & 4.754 & $1.76 \mathrm{E}-05$ & 8.49E-04 & $4 / 23$ & $\begin{array}{l}\text { AKT (PKB), G-protein } \alpha \text {-I family, } \\
\text { PKA-cat, PKA-reg }\end{array}$ \\
\hline 9 & $\begin{array}{l}\text { Development: leptin signaling via } \\
\text { PI3K-dependent pathway }\end{array}$ & 4.752 & $1.77 \mathrm{E}-05$ & 8.49E-04 & $5 / 47$ & $\begin{array}{l}\text { AKT (PKB), AKT2, CPT-1A, } \\
\text { PKA-cat, PKA-reg }\end{array}$ \\
\hline 10 & Apoptosis and survival: HTR1A signaling & 4.619 & $2.40 \mathrm{E}-05$ & $1.04 \mathrm{E}-03$ & $5 / 50$ & $\begin{array}{l}\text { AKT (PKB), G-protein a-I family, } \\
\text { PKA-cat, PKA-reg, PP2A regulatory }\end{array}$ \\
\hline
\end{tabular}

changes were specifically elicited by cross-fostering bLR offspring to bHR mothers, since fostering bLR offspring to bLR mothers produced minimal gene expression changes. It is also notable that most of our behavioral findings were specifically elicited by cross-fostering, with only 1 instance when bLR offspring displayed altered be- havior regardless of whether they were fostered to a bHR or bLR foster mother. In this case, bLR offspring that were raised by either a bHR or bLR foster mother showed less grooming in the NSF compared to bLR controls that were raised by their biological bLR mothers. Since we found no 'general fostering'-induced gene expression changes in 
the hippocampus or amygdala, it suggests that these effects on NSF behavior are mediated by molecular changes in another brain region.

Most neurodevelopmental milestones such as neurogenesis and neuronal migration occur in the amygdala well before P21. For instance, peak neurogenesis occurs at gestational days 11-16 within the major nuclei of the amygdala (basolateral, medial and central nuclei) [39], and neurons continue to migrate to the amygdala until P7 [40]. During the second postnatal week the total number of cells and the cell density of the amygdala decrease and remain stable after P14 [40]. The observed gene expression differences at P21 and P75 may be secondary to cross-fostering-induced structural changes in the amygdala. Future experiments could investigate changes in amygdalar structure and synaptic connectivity that may be driven by maternal care as well as those that exist at baseline in bHR versus bLR rats.

Our transcriptome analysis included GO, partitional clustering and functional pathway analyses to seek out ensembles of genes that were changed in the developing amygdala of bLR offspring cross-fostered to bHR mothers. A somewhat surprising finding was that, in general, the same genes were not altered across ages, suggesting that different molecular paths are engaged at different developmental stages. The partitional clustering analysis focused on the P75 time point since this was the age when the greatest numbers of genes were altered in the amygdala and was the time when social behavioral differences were apparent. Two of four gene clusters altered by cross-fostering were enriched with transcripts associated with cognition, learning, memory, and vesicle-mediated transport (fig. 3c). Pathway analysis also identified molecules involved in neural signaling and apoptotic processes as being changed by cross-fostering (table 2). Multiple differentially expressed genes were confirmed with qRT-PCR as upregulated in the amygdala of adult bLR offspring that were raised by bHR foster mothers versus biological bLR dams (Ywhag, Nptxr, CamkIIa, Sap-102, Psd-95, and Lppr2). Notably, these cross-foster-induced gene expression changes were observed in animals that were naïve to behavior testing. We typically perform gene expression analyses in untested animals to protect against the possibility that behavior testing itself may alter brain circuits and/or gene expression. However, it would be interesting to correlate behavior with gene expression and also to determine whether any of our 'top hit' genes are themselves altered by behavior tests. Future experiments could investigate these possibilities.
The gene clusters and pathways identified here are similar to a previous report that early unpredictable trauma triggered increased rodent anxiety-like behavior and adult amygdalar gene expression changes, including transcripts related to synaptic transmission, learning and memory [41]. Our findings are also congruent with studies linking anxiety-like behavior with synapse-associated proteins PSD-95 and SAP-102, which are members of the membrane-associated guanylate kinase protein family that regulate NMDA receptor signaling [42]. Genetic manipulation of PSD-95 increases anxiety-like behavior of rodents, alters social behavior and changes spine morphology in the amygdala $[43,44]$. PSD-95 and SAP-102 may also affect anxiety-like behavior through interactions with NMDA receptors in the amygdala since NMDA receptor activation is required for fear learning [45]. CamkIIa was another gene that emerged in our microarray data; it is a downstream target related to NMDA receptor activation and may also play a role in influencing anxiety and social behavior [42]. Future studies will investigate cross-fostering-induced synaptic changes in the bLR amygdala and interrogate roles of the specific genes identified here that may underlie the beneficial effect of cross-fostering on anxiety, and particularly social behavior, of bLRs.

Extensive work by Meaney et al. [21] demonstrates that Long-Evans rats raised by dams displaying low levels of active maternal care (licking/grooming and arched back nursing, LG-ABN) display greater anxiety-like behavior and exaggerated HPA stress responses compared to offspring of mothers showing high levels of LG-ABN $[21,46,47]$. In the high/low LG-ABN model, cross-fostering offspring from high to low LG-ABN dams (and vice versa) completely reversed behavioral and neuroendocrine effects [48] - in part through molecular changes in the hippocampus [47] and amygdala [46, 49]. This compelling work suggests that more maternal care begets better outcomes for offspring [21], although the relationship between maternal care and the biobehavioral stress response of offspring is more complex [6]. Our work and others suggest that the impact of maternal behavior on the behavior of offspring is dependent on the rat strain [19]. For example, our work in the bHR/bLR model (Sprague-Dawley rats) shows that bLR dams exhibit more active maternal care than bHR dams, yet their offspring display higher levels of anxiety/depression-like behavior $[19,20]$. In the context of the present study, it is important to note that we previously showed that cross-fostering does not alter the behavior of bHR/bLR mothers [20]. Moreover, examples from human [50] and nonhuman 
primate [51] studies show that 'overprotective' mothers can contribute to excessive shyness and timidity in offspring, which could be considered analogous to the highly maternal bLR dams and their inhibited bLR offspring. In any event, our current cross-fostering data indicate that the distinct maternal style of bHR dams benefits bLR offspring relative to bLRs raised by their own biological bLR mothers or bLR foster mothers.

A limitation of the current study is the lack of female offspring included in the behavioral and gene expression experiments. It would be interesting to determine whether cross-fostering bLR female offspring also impacts their behavior (whether anxiety behavior, social behavior or even maternal behavior). Work by Meaney and colleagues [48] has shown that cross-fostering female offspring born to low LG-ABN mothers to high LG-ABN dams converts their later patterns of maternal care so that females behave in a manner that follows the maternal care they received. It would be interesting to determine whether cross-fostering bLR females to bHR mothers would similarly alter their later maternal care.

In summary, cross-fostering high anxiety/depressionprone bLR rats to bHR mothers shifted developmental gene expression in the amygdala (but not the hippocampus) and modified their adult phenotype by enhancing their typically low levels of social interaction and subtly improving their anxiety-like behavior. In spite of the strong genetic underpinnings of the bHR/bLR traits, our current findings and other recent work demonstrate how early-life factors influence bLR behavior [14]. Moreover, while earlier studies highlighted the importance of hippocampal differences contributing to the disparate bHR/ bLR behavioral phenotypes $[18,33]$, our present results point to a role of the amygdala as well. Future work will pursue molecular and cellular mechanisms within the amygdala that contribute to bHR/bLR behavioral differences either at baseline or following early-life environmental manipulations.

\section{Acknowledgments}

We are very grateful to Nateka Jackson and Sara Anne Stringfellow for their excellent technical assistance.

\section{Disclosure Statement}

The study was funded by NIMH 4R00MH085859-02 (S.M.C.), NIGMS MSTP T32GM008361, Office of Naval Research ONRN00014-09-1-0598 (H.A.) and NIDA PPG 5P01DA021633-02 (H.A.).

The authors declare no conflicts of interest.

\section{References}

1 Caspi A, Sugden K, Moffitt TE, Taylor A, Craig IW, Harrington H, McClay J, Mill J, Martin J, Braithwaite A, Poulton R: Influence of life stress on depression: moderation by a polymorphism in the 5-HTT gene. Science 2003;301:386-389.

2 Kim-Cohen J, Moffitt TE, Taylor A, Pawlby SJ, Caspi A: Maternal depression and children's antisocial behavior: nature and nurture effects. Arch Gen Psychiatry 2005;62:173-181.

- 3 Halligan SL, Murray L, Martins C, Cooper PJ: Maternal depression and psychiatric outcomes in adolescent offspring: a 13-year longitudinal study. J Affect Disord 2007;97:145-154.

4 Weissman MM, Jensen P: What research suggests for depressed women with children. J Clin Psychiatry 2002;63:641-647.

5 Herba CM, Tremblay RE, Boivin M, Liu X, Mongeau C, Seguin JR, Cote SM: Maternal depressive symptoms and children's emotional problems: can early child care help children of depressed mothers? JAMA Psychiatry 2013;70:830-838.

-6 Tang AC, Reeb-Sutherland BC, Romeo RD, McEwen BS: On the causes of early life experience effects: evaluating the role of mom. Front Neuroendocrinol 2014;35:245-251.
7 Moffitt TE, Harrington H, Caspi A, Kim-Cohen J, Goldberg D, Gregory AM, Poulton R: Depression and generalized anxiety disorder: cumulative and sequential comorbidity in a birth cohort followed prospectively to age 32 years. Arch Gen Psychiatry 2007;64:651-660.

-8 Stead JD, Clinton S, Neal C, Schneider J, Jama A, Miller S, Vazquez DM, Watson SJ, Akil H: Selective breeding for divergence in noveltyseeking traits: heritability and enrichment in spontaneous anxiety-related behaviors. Behav Genet 2006;36:697-712.

-9 Kerman IA, Clinton SM, Bedrosian TA, Abraham AD, Rosenthal DT, Akil H, Watson SJ: High novelty-seeking predicts aggression and gene expression differences within defined serotonergic cell groups. Brain Res 2011;1419:34-45.

10 Flagel SB, Waselus M, Clinton SM, Watson SJ, Akil $\mathrm{H}$ : Antecedents and consequences of drug abuse in rats selectively bred for high and low response to novelty. Neuropharmacology 2014;76 Pt B:425-436.

1 Clinton S, Miller S, Watson SJ, Akil H: Prenatal stress does not alter innate novelty-seeking behavioral traits, but differentially affects individual differences in neuroendocrine stress responsivity. Psychoneuroendocrinology 2008;33:162-177.

12 Stedenfeld KA, Clinton SM, Kerman IA, Akil $\mathrm{H}$, Watson SJ, Sved AF: Novelty-seeking behavior predicts vulnerability in a rodent model of depression. Physiol Behav 2011;103:210-216.

13 Garcia-Fuster MJ, Parks GS, Clinton SM, Watson SJ, Akil H, Civelli O: The melanin-concentrating hormone $(\mathrm{MCH})$ system in an animal model of depression-like behavior. Eur Neuropsychopharmacol 2012;22:607-613.

14 Clinton SM, Watson SJ, Akil H: High noveltyseeking rats are resilient to negative physiological effects of the early life stress. Stress 2014;17:97-107.

15 Charlesworth B: Fundamental concepts in genetics: effective population size and patterns of molecular evolution and variation. Nat Rev Genet 2009;10:195-205.

16 Kabbaj M: Individual differences in vulnerability to drug abuse: the high responders/low responders model. CNS Neurol Disord Drug Targets 2006;5:513-520.

-17 Blanchard MM, Mendelsohn D, Stamp JA: The HR/LR model: further evidence as an animal model of sensation seeking. Neurosci Biobehav Rev 2009;33:1145-1154. 
-18 Clinton SM, Stead JD, Miller S, Watson SJ, Akil $\mathrm{H}$ : Developmental underpinnings of differences in rodent novelty-seeking and emotional reactivity. Eur J Neurosci 2011;34:994-1005.

-19 Clinton SM, Vazquez DM, Kabbaj M, Kabbaj $\mathrm{MH}$, Watson SJ, Akil H: Individual differences in novelty-seeking and emotional reactivity correlate with variation in maternal behavior. Horm Behav 2007;51:655-664.

20 Clinton SM, Bedrosian TA, Abraham AD, Watson SJ, Akil H: Neural and environmental factors impacting maternal behavior differences in high- versus low-novelty-seeking rats. Horm Behav 2010;57:463-473.

21 Meaney MJ: Maternal care, gene expression, and the transmission of individual differences in stress reactivity across generations. Ann Rev Neurosci 2001;24:1161-1192.

-22 Nam H, Clinton SM, Jackson NL, Kerman IA: Learned helplessness and social avoidance in the Wistar-Kyoto rat. Front Behav Neurosci 2014;8:109.

-23 Nin MS, Couto-Pereira NS, Souza MF, Azeredo LA, Ferri MK, Dalpra WL, Gomez R, Barros HM: Anxiolytic effect of clonazepam in female rats: grooming microstructure and elevated plus maze tests. Eur J Pharmacol 2012; 684:95-101.

24 Denmark A, Tien D, Wong K, Chung A, Cachat J, Goodspeed J, Grimes C, Elegante M, Suciu C, Elkhayat S, Bartels B, Jackson A, Rosenberg M, Chung KM, Badani H, Kadri F, Roy S, Tan J, Gaikwad S, Stewart A, Zapolsky I, Gilder T, Kalueff AV: The effects of chronic social defeat stress on mouse self-grooming behavior and its patterning. Behav Brain Res 2010;208:553-559.

25 Archer J: Tests for emotionality in rats and mice: a review. Anim Behav 1973;21:205-235.

26 Walsh RN, Cummins RA: The open-field test: a critical review. Psychol Bull 1976;83:482-504.

-27 Simmons RK, Stringfellow SA, Glover ME, Wagle AA, Clinton SM: DNA methylation markers in the postnatal developing rat brain. Brain Res 2013;1533:26-36.

-28 Heim C, Newport DJ, Wagner D, Wilcox MM, Miller AH, Nemeroff CB: The role of early adverse experience and adulthood stress in the prediction of neuroendocrine stress reactivity in women: a multiple regression analysis. Depress Anxiety 2002;15:117-125.

-29 Apter-Levy Y, Feldman M, Vakart A, Ebstein RP, Feldman R: Impact of maternal depression across the first 6 years of life on the child's mental health, social engagement, and empathy: the moderating role of oxytocin. Am J Psychiatry 2013;170:1161-1168.
30 Kessler MS, Bosch OJ, Bunck M, Landgraf R, Neumann ID: Maternal care differs in mice bred for high vs. low trait anxiety: impact of brain vasopressin and cross-fostering. Soc Neurosci 2011;6:156-168.

-31 Schroeder M, Weller A: Anxiety-like behavior and locomotion in CCK1 knockout rats as a function of strain, sex and early maternal environment. Behav Brain Res 2010;211:198207.

32 Kaffman A, Krystal JH: New frontiers in animal research of psychiatric illness. Methods Mol Biol 2012;829:3-30.

33 Turner CA, Clinton SM, Thompson RC, Watson SJ Jr, Akil H: Fibroblast growth factor-2 (FGF2) augmentation early in life alters hippocampal development and rescues the anxiety phenotype in vulnerable animals. Proc Natl Acad Sci U S A 2011;108:80218025

-34 Allsop SA, Vander Weele CM, Wichmann R, Tye KM: Optogenetic insights on the relationship between anxiety-related behaviors and social deficits. Front Behav Neurosci 2014;8:241.

35 Felix-Ortiz AC, Beyeler A, Seo C, Leppla CA, Wildes CP, Tye KM: BLA to VHPC inputs modulate anxiety-related behaviors. Neuron 2013;79:658-664.

36 Tye KM, Prakash R, Kim SY, Fenno LE, Grosenick L, Zarabi H, Thompson KR, Gradinaru V, Ramakrishnan C, Deisseroth K: Amygdala circuitry mediating reversible and bidirectional control of anxiety. Nature 2011; 471:358-362.

37 Felix-Ortiz AC, Tye KM: Amygdala inputs to the ventral hippocampus bidirectionally modulate social behavior. J Neurosci 2014;34: 586-595.

38 Hong W, Kim DW, Anderson DJ: Antagonistic control of social versus repetitive selfgrooming behaviors by separable amygdala neuronal subsets. Cell 2014;158:1348-1361.

-39 Berger MA, Barros VG, Sarchi MI, Tarazi FI, Antonelli MC: Long-term effects of prenatal stress on dopamine and glutamate receptors in adult rat brain. Neurochem Res 2002;27: 1525-1533.

40 Berdel B, Morys J, Maciejewska B: Neuronal changes in the basolateral complex during development of the amygdala of the rat. Int J Dev Neurosci 1997; 15:755-765.

41 Sarro EC, Sullivan RM, Barr G: Unpredictable neonatal stress enhances adult anxiety and alters amygdala gene expression related to serotonin and GABA. Neuroscience 2014;258: 147-161.
42 Fan X, Jin WY, Wang YT: The NMDA receptor complex: a multifunctional machine at the glutamatergic synapse. Front Cell Neurosci 2014;8:160.

43 Feyder M, Karlsson RM, Mathur P, Lyman M, Bock R, Momenan R, Munasinghe J, Scattoni ML, Ihne J, Camp M, Graybeal C, Strathdee D, Begg A, Alvarez VA, Kirsch P, Rietschel M, Cichon S, Walter H, Meyer-Lindenberg A, Grant SG, Holmes A: Association of mouse Dlg4 (PSD-95) gene deletion and human DLG4 gene variation with phenotypes relevant to autism spectrum disorders and Williams' syndrome. Am J Psychiatry 2010;167: 1508-1517.

44 Nagura H, Ishikawa Y, Kobayashi K, Takao K, Tanaka T, Nishikawa K, Tamura H, Shiosaka S, Suzuki H, Miyakawa T, Fujiyoshi Y, Doi T: Impaired synaptic clustering of postsynaptic density proteins and altered signal transmission in hippocampal neurons, and disrupted learning behavior in PDZ1 and PDZ2 ligand binding-deficient PSD-95 knockin mice. Mol Brain 2012;5:43.

-45 Gupta-Agarwal S, Jarome TJ, Fernandez J, Lubin FD: NMDA receptor- and ERK-dependent histone methylation changes in the lateral amygdala bidirectionally regulate fear memory formation. Learn Mem 2014;21: 351-362.

46 Caldji C, Tannenbaum B, Sharma S, Francis D, Plotsky PM, Meaney MJ: Maternal care during infancy regulates the development of neural systems mediating the expression of fearfulness in the rat. Proc Natl Acad Sci U S A 1998; $95: 5335-5340$

47 Weaver IC, Meaney MJ, Szyf M: Maternal care effects on the hippocampal transcriptome and anxiety-mediated behaviors in the offspring that are reversible in adulthood. Proc Natl Acad Sci U S A 2006; 103:3480-3485.

48 Francis D, Diorio J, Liu D, Meaney MJ: Nongenomic transmission across generations of maternal behavior and stress responses in the rat. Science 1999;286:1155-1158.

49 Caldji C, Diorio J, Meaney MJ: Variations in maternal care alter GABA(A) receptor subunit expression in brain regions associated with fear. Neuropsychopharmacology 2003; 28:1950-1959.

50 Cooper PJ, Eke M: Childhood shyness and maternal social phobia: a community study. Br J Psychiatry 1999;174:439-443.

51 Fairbanks LA, McGuire MT: Long-term effects of early mothering behavior on responsiveness to the environment in vervet monkeys. Dev Psychobiol 1988;21:711-724. 